\title{
Brief interventions for cannabis use in emerging adults: protocol for a systematic review, meta-analysis, and evidence map
}

\author{
Jillian Halladay ${ }^{1,2,3}$, Tashia Petker ${ }^{3,4}$, Allan Fein ${ }^{2,3}$, Catharine Munn ${ }^{2,3,4}$ and James MacKillop ${ }^{2,3,4^{*}}$ (D)
}

\begin{abstract}
Background: Rates of cannabis use are highest during emerging adulthood (age 18-25), with the prevalence of near daily and daily increasing among this age group. Emerging adults are clinically challenging in terms of harmful cannabis use due to perceptions of high rates of peer use, social acceptance, and low risk of harm. Brief interventions to increase awareness and promote motivation to change are therefore particularly important for this age group. There is existing evidence on the effectiveness of brief interventions for alcohol in emerging adults, but it is not clear if comparable evidence is present for cannabis. The objective of this systematic review is to summarize and critically appraise the existing literature of brief interventions for cannabis use both narratively, to describe the content and delivery of existing interventions, and meta-analytically, to determine the aggregated efficacy of these interventions on cannabis use and other outcomes (e.g., other substance use, mental health, help-seeking behaviors, and academic and occupational outcomes).

Methods: A systematic search of randomized controlled trials, quasi-experimental trials, and pre-post designs will be conducted in the following electronic databases: MEDLINE, EMBASE, Cochrane Central Register of Controlled Trials, Allied and Complementary Medicine, Cumulative Index to Nursing and Allied Health Literature, and PsycINFO. Ongoing trials will be identified using the World Health Organization International Clinical Trials Registry Platform, ClinicalTrials.gov, and Current Controlled Trials. Unpublished trials will be identified using Proquest Dissertations, OpenGrey, Google Scholar, and brief interventions on the Substance Abuse and Mental Health Services Administration webpage. Two authors will independently screen and extract data from articles using a predetermined screening and extraction forms (which will include risk of bias assessments). Calibration exercises will be performed prior to full screening and extraction. Disagreements will be resolved through discussion or consultation with a third reviewer. All studies will be reported narratively, and if appropriate, we will perform random effects meta-analyses with subgroup analyses and meta-regression.

Discussion: Results of this review are expected to provide guidance on the content, delivery methods, and effectiveness of brief interventions for cannabis use to assist post-secondary institutions in identifying brief intervention strategies to implement prior to or in response to legalization.
\end{abstract}

Systematic review registration: CRD42018085412

Keywords: Cannabis, Marijuana, Brief intervention, Motivational interviewing, Motivational enhancement, Emerging adult, Student, Meta-analysis, Systematic review

\footnotetext{
* Correspondence: jmackill@mcmaster.ca

${ }^{2}$ Michael G. De Groote Centre for Medicinal Cannabis Research, McMaster

University/St. Joseph's Healthcare Hamilton, Hamilton, Ontario, Canada

${ }^{3}$ Peter Boris Centre for Addictions Research, McMaster University/St. Joseph's

Healthcare Hamilton, 100 West 5th Street, Hamilton, Ontario L8S 4L8, Canada

Full list of author information is available at the end of the article
}

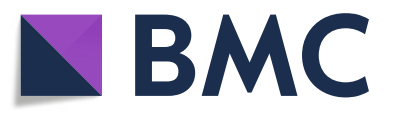

(c) The Author(s). 2018 Open Access This article is distributed under the terms of the Creative Commons Attribution 4.0 International License (http://creativecommons.org/licenses/by/4.0/), which permits unrestricted use, distribution, and reproduction in any medium, provided you give appropriate credit to the original author(s) and the source, provide a link to the Creative Commons license, and indicate if changes were made. The Creative Commons Public Domain Dedication waiver (http://creativecommons.org/publicdomain/zero/1.0/) applies to the data made available in this article, unless otherwise stated. 


\section{Background}

Cannabis is one of the most commonly used psychoactive substances in the world, with use most prevalent among emerging adults (15-24 year olds) [1-5]. Cannabis use has been associated with negative physical and mental health effects in a dose-response fashion, where near daily or daily use is associated with worse outcomes [6]. Harmful levels of use have been increasing among emerging adults in Canada [7, 8] and the United States [1]. Risks of cannabis-related harm are more pervasive for individuals who begin using cannabis during adolescence, with earlier age of first use potentially resulting in long lasting consequences [6]. Given the high prevalence of use and potential for significant impairment, efficacious interventions for high-risk individuals in this age group are a pressing clinical priority.

Emerging adults are neurodevelopmentally vulnerable to the effects of cannabis, as cannabis acts on areas of the brain integral to brain development. The brain is not completely matured until approximately 25 years of age [9]. Chronic, regular cannabis use during adolescence has shown to negatively affect memory, attention, and psychomotor skills [10-12], potentially causing irreversible cognitive impairment [13] resulting in an increased likelihood of fatal car accidents [14], poor academic performance [15-17], and dropping out of school [17] . Additionally, weekly cannabis use or a cannabis use disorder is almost 10 times as likely in people with other mental illness as compared to those without other mental illness [18]; frequent use has been associated with psychotic disorders [14, 19], bipolar disorder [14], personality disorders [20], depression [20-22], anxiety [23], and suicidal ideation and attempts [24]. Overall, cannabis use during emerging adulthood is particularly concerning due to its potential to disrupt neurological, social, emotional, and cognitive development.

Despite the associations between cannabis use and negative health consequences, the perceived risk of using cannabis, both occasionally and regularly, is decreasing among adolescents and emerging adults [25-28] and, subsequently, there has been an increase in social acceptance of cannabis use [29]. This is further compounded by the perception of high-frequency peer use; in North America, emerging adults perceive that about $86 \%$ of their peers are using cannabis at least monthly when only about $20 \%$ of students actually report using monthly [7, 30]. Additionally, for general mental health concerns, less than half of emerging adults in need receive professional help [31] with common reported barriers to treatment including stigma, embarrassment, problems recognizing symptoms, self-reliance, and importantly, not enough time [32, 33]. These barriers to seeking general mental health services may be magnified for substance use concerns due to the perception of normalcy of problematic behaviors. Therefore, the perception of low-risk, high peer use, and social acceptance make emerging adults a clinically challenging population, which is further compounded by low motivation for change.

Existing literature on interventions targeting post-secondary substance users acknowledges the challenges to engaging this population and, therefore, has focused on brief interventions (BI) or brief motivational interventions (BMIs). There are many different existing definitions for brief interventions but all contain typical ingredients. BIs typically are short in duration $[34,35]$ and include provision of personalized feedback [36]. Most BIs adopt a motivational interviewing (MI) approach with the primary goal of the BI being to motivate participants to change their behavior, teach behavioral change skills, and connect to services [35, 37-39]. Often, BIs follow the FRAMES model in which the participant receives personalized feedback, reinforcement of personal responsibility, objective advice given non-judgmentally, a menu of options, an empathetic and accepting listener, and encouragement of self-efficacy and confidence [38-40].

Emerging adulthood is a distinct developmental period characterized by instability and exploration, often referred to as extended adolescence, in which individuals typically experience reduced parental monitoring but are not yet fully engaged in the responsibilities and expectations of adulthood [41]. Emerging adults straddle adolescence and young adulthood, sharing some similarities with both developmental periods. A scoping search was performed to identify the general breadth of existing brief intervention literature to inform the specifics of this review. Existing literature on BIs for cannabis use is already limited, and is particularly scarce when focused on emerging adults. Thus, BIs for adolescents and young adults should also be explored to provide a comprehensive picture and clinical relevance of the existing $\mathrm{BI}$ literature.

We identified three reviews on BIs for adolescent substance use. Jensen (2011) looked specifically at MI approaches (including BIs and longer duration interventions, 1 to 9 sessions) for adolescent (12-20 years of age) substance use, finding evidence that suggests MI approaches are helpful to reduce general substance use in the short and longer term (significance retained at follow-up) [42]. Barnett (2012) conducted a systematic review on MI interventions for adolescent substance use (mean age $<18.5$ ). Although they did not perform a meta-analysis, they indicate that $67 \%$ of the studies result in reductions in some form of substance use outcomes, including marijuana use [43]. A Cochrane review by Carney (2016) found six randomized controlled trials (RCTs) of face-to-face school-based BIs for adolescents (under 19 years of age) who experience negative behavioral consequences from subclinical levels of substance use [44]. Moderate-quality evidence suggests that effects of BIs on 
cannabis frequency and dependence are not significantly different compared to information provision (health promotion materials and harm reduction information). However, when comparing BIs to assessment only (evaluated on substance use but received no intervention), BIs appear to reduce cannabis frequency (SMD - 0.54 [- 0.77, - 0.31]), although this is based on low-quality evidence and only includes results from two RCTs $(n=338)$.

For emerging adults, we identified one relevant literature review by Dennhardt and colleagues (2013) [45]. This review found one observational study and five RCTs on BIs and examined their effect on substance use outcomes, including cannabis use. They did not perform a systematic search and did not combine results meta-analytically, but concluded that brief motivational interventions demonstrated the most promising results for college students, apart from parent-based interventions, and requires further investigation.

For adults, there is a Cochrane review by Gates et al. (2016) on psychosocial interventions for cannabis use [46]. This study found 23 RCTs of psychosocial interventions (which include CBT, MI, mindfulness, counseling or education contingency management, relapse prevention) for adults with cannabis abuse or dependence or near daily users of cannabis who were seeking treatment for their cannabis use or other adults seeking treatment for cannabis use. Results suggest moderate-quality evidence that individuals who receive a psychosocial intervention use cannabis on fewer days compared to inactive control; low-quality evidence that those receiving an intervention were more likely to report point-prevalence abstinence, fewer symptoms of dependence, and fewer cannabis-related problems compared to inactive control; and very low-quality evidence that individuals receiving interventions use fewer joints per day compared with inactive control. Additionally, interventions longer than four sessions for more than a month appear to demonstrate better outcomes and Cognitive Behavioral Therapy (CBT) appears to produce the largest effects followed by motivational approaches.

There are several methodological and conceptual limitations of existing reviews. Methodologically, one study did not including a systematic search [45], two did not include unpublished literature [42, 43], and two only included RCTs [44, 46]. In regards to the interventions, most did not look at BIs (1-2 sessions) neither as a whole or within a subgroup analysis [42, 43, 46], most were not cannabis specific [42-45], and there were common restrictions based on the delivery location of interventions (i.e., only in secondary school [44], only in person [44, 46], no inpatient [42], only outpatient community [46]). In the two most comprehensive Cochrane reviews, there were participant restrictions based on baseline substance use; Gates required participants to have a minimum amount of cannabis use at baseline and excluded individuals who regularly used or had a substance dependence on a substance other than marijuana or nicotine [46] while Carney excluded any individuals who had any substance dependence [44]. Also, only one review covered a youth population (12-25) and is fraught with other methodologically and conceptual limitations including being outdated [42]. Additionally, the lack of gold standard BI approach for cannabis use means that there is significant variability in the content and delivery methods of existing BIs. This variability increases heterogeneity and limits our ability to determine the salient components of a successful BI.

This review seeks to address these gaps by (1) focusing primarily on cannabis related outcomes, (2) conducting an up-to-date systematic search of all BIs for cannabis, inclusive of all youth populations and study designs, (3) providing comprehensive descriptions of the contents and delivery methods of existing BIs, and (4) performing multiple subgroup analyses based on content and delivery methods to try to determine the most important and effective components of a BI.

\section{Objectives}

The research question for this review is as follows: In youth aged 15-24, what is the content and effect of existing brief interventions (1-2 sessions) for cannabis use on (a) cannabis-related outcomes, (b) other substance use, (c) help-seeking behaviors, (d) mental health and well-being, and (e) academic and occupational outcomes? Specifically, our aims are as follows:

1) Qualitatively summarize the content and delivery methods of existing BIs cannabis use

2) Summarize and evaluate the quality of existing evidence of cannabis BI for outcomes including (a) cannabis related outcomes, (b) other substance use, (c) mental health and well-being, and (d) academic and occupational outcomes

3) Quantitatively synthesize primary studies

4) Determine key components and delivery methods of successful brief interventions to assist in identifying the ingredients for a gold standard cannabis BI

5) Present an evidence map of BIs for cannabis use across developmental age groups to synthesize existing evidence in a user-friendly format and identify developmental age-specific gaps in the literature

\section{Methods}

Eligibility criteria

\section{Study designs}

We will include all RCT designs including parallel-group, crossover, cluster-randomized and factorial design trials, 
and observational studies including pre-post designs and quasi-randomized trials.

\section{Population}

The target population is emerging adults (i.e., 15-24 years of age) [5]. This will include individuals both in and outside of school. We will code studies based on context (e.g., secondary school, university, college, treatment, correctional, etc.). Acknowledging the paucity of literature, we will expand our search to any samples that overlap with our pre-identified emerging adult age group (e.g., adolescents age 13-19 or adults 18+). Studies with samples with a maximum age less than 15 and the minimum age greater than 24 will be excluded. This review will not place restrictions on samples based on nature of participation (i.e., voluntary, mandated), presence or absence of other mental health concerns, student status, study origin, ethnicity, or sexuality.

\section{Intervention}

Brief interventions will be operationalized as 1-2 sessions focused on cannabis use. BIs including FRAMES interventions are commonly defined as a single session. Including a second session constitutes a reasonable expansion of this definition and allows for scenarios in which an individual provides information at one session and receives feedback at a second session. Interventions comprising 3+ sessions will be excluded for falling outside the scope of being BIs. We will include BIs exclusively focused on cannabis use. BIs focused on cannabis use in combination with alcohol use, tobacco use, or general substance use will be excluded. We will include interventions that conduct an additional preliminary or follow-up session for the purpose of data collection and/or behavioral analysis. We will not place restrictions on method of delivery (i.e., in person, online, over the phone).

\section{Comparison}

Comparison groups can include within-participant pre-post data, wait list control, treatment as usual, or active controls. This will allow for the inclusion of single-arm, two-arm, or multi-armed randomized or observational trials.

\section{Outcome}

No gold standard instrument currently exists for the screening and assessment of cannabis use. Although there are 25 screening and assessment instruments available with adequate psychometric properties, evidence for the effectiveness of these tools is limited [47]. With this in mind, any instrument capturing aspects of cannabis use (e.g., frequency, problems, dependence, motivation to change) will be included in this systematic review for cannabis use outcomes. We will also collect data on secondary outcomes including all other substance use outcomes, help-seeking behaviors (e.g., accessing services), mental health (both self-report and diagnostic outcomes), and any academic or occupational related outcomes. Data will be collected for all follow-up time points available.

\section{Search strategy}

We will search OVID MEDLINE In-Process (1946 to present), EMBASE (1974 to present), the Cochrane Central Register of Controlled Trials (CENTRAL), Allied and Complementary Medicine (AMED 1985 to present), CINAHL, and PsychInfo (1806 to present). Ongoing trials will be identified using the World Health Organization (WHO) International Clinical Trials Registry Platform, Clinical Trials.gov, and Current Controlled Trials (http://www.controlled-trials.com/). Unpublished trials will be identified using Proquest Dissertations, OpenGrey (up to February 2017), Google Scholar (1st 50 hits for each search), and brief interventions on the Substance Abuse and Mental Health Services Administration (http://nrepp.samhsa.gov/). No restrictions will be placed on language or publication status. We will check abstracts and reference lists of included articles and existing systematic reviews and contact authors for further information and data when appropriate (two attempts will be made). See Additional file 1 for full search strategy in each database and initial hits for each search (will re-run search to ensure up to date).

\section{Data collection}

\section{Selection of studies}

Articles will be uploaded to Covidence, an online systematic review management software. Two review authors will use Covidence to screen titles and abstracts of identified studies for possible inclusion based on screening forms (see Additional file 2). We will perform a calibration exercise to maximize consistency ensuring good inter-rater reliability operationalized as a Cohen's kappa of 0.8. After title and abstract screening, all studies that are selected for full-text review (without regard for agreement between screeners) will be screened. Again, two reviewers will independently assess full-texts for inclusion and disagreements will be resolved through discussion or consultation with a third reviewer. Following completion of full-text screening, final inter-rater reliability will be calculated.

\section{Data extraction and management}

One review author will independently extract data to a Microsoft Excel spreadsheet including study ID, study design, demographic data, risk of bias assessment, intervention description, comparison description, outcomes and measurement tools, summary of author's findings, and other comments (see Additional file 3 for full extraction content). A second review author will verify the 
extraction. A calibration exercise will be performed to maximize consistency. Discrepancies will be resolved through discussion or consultation with a third reviewer.

\section{Risk of bias of included studies}

Risk of bias (RoB) of included RCTs will be assessed using the Cochrane RoB tool at the study level [48]. Each domain will be judged as "low" or "high" RoB and reviewers will avoid judging bias as "unclear." Observational studies will be assessed with the RoB in non-randomized studies of interventions (ROBINS-I) tool [49]. We will also include the presence of intervention fidelity checks in our RoB assessments under "other" in Cochrane RoB for RCTs and under "bias due to deviations from intended interventions" in ROBINS-I tool for observational studies. Two review authors will independently conduct RoB assessments. Discrepancies in judgments will be discussed and, if a consensus is not met, a third reviewer will resolve the remaining discrepancies.

\section{Data synthesis, interpretation, and presentation}

We will be collecting continuous and dichotomous outcomes, regardless of the measurement tool. We will use mean differences when continuous outcomes are measured similarly (e.g., frequency of cannabis use, motivation to change, academic grades). Standardized mean differences (SMDs) will be used to combine continuous outcomes of similar constructs measured with different instruments (e.g., measures of substance use or mental health symptoms severity). SMDs will be calculated using the inverse variance method in a random effects model to create a uniform measurement scale and allow pooling of effects across studies. Review Manager 5 (RevMan) software will be used to calculate SMDs and 95\% confidence intervals (CI), which uses the Hedges' adjusted g formula [48]. SMDs (Hedges' g) of 0.2, 0.5, and 0.8 are interpreted as small, medium, and large effects respectively $[48,50]$. We will adjust for factorial designs if a particular group (i.e., control) needs to be entered into the same meta-analysis twice by dividing the sample size of the duplicate group in half, keeping means and standard deviations unchanged [48]. For cluster-randomized studies, the data will be adjusted for the clustering effect by using either the reported adjusted post-score means or the intra-class correlation coefficient (ICC) to calculate the associated design effect (DE) and effective sample size [48].

For dichotomous outcomes (e.g., presence or absence of substance or other mental disorder, access of follow-up services, employment or student status), relative risks (RR) and odds ratios (OR) will be combined using the inverse variance method in a random effects model and reported as either a pooled RR or OR (depending on what is more commonly available in the existing literature) with subgroup analyses comparing ORs and RRs to ensure it is appropriate to pool measures [48]. If pooling results is not possible due to insufficient studies (i.e., less than 2), results will be presented narratively.

\section{Dealing with missing data}

When there is missing data, trialists will be contacted to obtain missing information (two attempts will be made). If we do not hear from trialists, we will calculate or impute missing values. Missing standard deviations (SDs) will be algebraically calculated using standard error, 95\% confidence intervals, or $p$ values. If missing SDs cannot be calculated algebraically, and baseline and final SDs are known, SDs will be imputed using a correlation coefficient or a conservative estimate of 0.5 . We will also perform a sensitivity analysis for studies that only provide complete case data compared to studies that follow intention-to-treat principles. For dichotomous data, if events are missing, we will perform sensitivity analyses considering worst case scenario (i.e., assuming all participants experienced the "bad" outcome) and best case scenario (i.e., assuming all participants experienced the "good" outcome).

\section{Assessment of heterogeneity}

Heterogeneity between trials pooled for meta-analyses will be assessed by (1) visual inspection of the forest plot, (2) $\mathrm{X}^{2}$ test for statistically significance $(P<0.1)$, and $(3) \mathrm{I}^{2}$ statistic to examine the proportion of between trial differences not due to chance [48]. The $\mathrm{I}^{2}$ statistic will be used to determine the proportion of differences that was not due to chance, where values were assessed as 'might not be important' (0\%-40\%), “moderate" (30\%-60\%), "substantial" (50\%-90\%), or "high" (75\% to $100 \%)$ [48]. Due to the overlap in classification of $\mathrm{I}^{2}$ categories, a moderate $\mathrm{I}^{2}$ in our study will be operationalized as $40 \%$ due to the fact that some degree of heterogeneity would be expected between behavioral interventions. Where subgroup analyses have a minimum of 10 studies in the smaller subgroup, we will carry out meta-regression to test subgroup analyses, and multiple meta-regressions (sample size permitting). All pre-specified subgroup analyses will be carried out regardless of heterogeneity.

\section{Subgroup analyses and sensitivity analysis}

Gates (2016) conducted a fairly comprehensive set of subgroup analyses for psychosocial interventions targeting cannabis use. We will include all subgroup analyses Gates performed with some additional tests: (1) length of the intervention; (2) method of delivery (i.e., in-person compared to online or over the phone interventions); (3) population characteristics including (a) proportion of males, (b) clinical characteristics (dependence, concurrent other psychiatric illness, concurrent non-cannabis 
substance use, healthy), (c) treatment-seeking compared to non-treatment-seeking participants (including voluntary compared to mandatory participation), (d) patterns of cannabis use history (e.g., duration or levels of use, number of days of use, number of uses per day, age of initiation, route of delivery); (4) content characteristics including (a) personalized feedback regarding frequency, (b) personalized feedback regarding other outcomes, (c) normative feedback, (d) pros and cons, (e) values exercises, (f) mental health screening and/or discussion, (g) decision balance and/or goal setting, (h) provision of additional resource, and (i) adjunctive therapy or booster sessions; (5) study quality (low and high RoB); (6) study design (RCT and observational studies); and for dichotomous outcomes, (7) RR compared to OR, and (8) measurement instrument used. Significant subgroup differences will be identified using a $p$ value of $<0.1$. All subgroup topics will also be reported descriptively. If enough studies are present, univariate and multivariate meta-regression analyses will be performed. If possible, all analyses will be performed on the total sample of available studies as well as stratified by developmental age (e.g., adolescents, emerging adults, adults).

\section{Quality assessment and presentation}

The Grading or Recommendations Assessment, Development, and Evaluation (GRADE) approach will be used to assess the quality of the evidence for each outcome to help infer confidence in the review findings and guide future research [51]. Pooled SMD estimates or pooled RR/ORs, along with the GRADE assessment, will be presented in a Summary of Findings table. This protocol follows procedures outlined in the Preferred Reporting Items for Systematic Reviews and Meta-Analysis Protocols (PRISMA-P) statement (see Additional file 4).

We will additionally create an evidence map using a cross-tabular format of BIs for cannabis use across age groups (e.g., adolescents, emerging adults, and adults) to organize the evidence. Miake-Lye et al. (2016) identified cross-tabular formats as the most common way to present evidence "maps" [52]. This evidence map will detail the number and quality of RCTs and observational studies, the combined sample size, the outcomes measured, and the content of interventions stratified by developmental age group.

\section{Discussion}

Results of this systematic review will help to guide the development and refinement of brief interventions targeting cannabis use in the emerging adult population. Identifying the components of effective interventions is critical to inform brief interventions, which can engage youth and promote further help-seeking and behavior change. Given the potential for long-lasting negative effects of cannabis use during emerging adulthood, and current and impending changes in decriminalization and legalization of recreational cannabis in North America, this is an important step in reducing cannabis use and harms. Brief interventions may hold significant potential in terms of providing cost-effective, time-effective, and youthfriendly interventions. This review is essential to help inform effective BI practices that warrant implementation into practice will guide informed future investigations.

\section{Additional files}

Additional file 1: Search strategy. Completed search strategy corresponding with this protocol. (PDF $61 \mathrm{~kb}$ )

Additional file 2: Screening form. First draft of the screening form (not yet piloted). (PDF $56 \mathrm{~kb}$ )

Additional file 3: Data extraction content. Data that will be extracted from each included full text in this review. (DOCX $92 \mathrm{~kb}$ )

Additional file 4: PRISMA-P checklist. Completed PRISMA-P checklist applied to this protocol. (PDF $165 \mathrm{~kb}$ )

\section{Abbreviations}

BI: Brief intervention; CBT: Cognitive behavioral therapy; Cl: Confidence interval; GRADE: Grading or recommendations assessment, development, and evaluation; MET: Motivational enhancement therapy; MI: Motivational interviewing; RoB: Risk of bias; ROBINS-I: Risk of bias in non-randomized studies of interventions; SMD: Standardized mean difference

\section{Acknowledgements}

Mr. Justin Scherer, Ms. Vanessa Linton, Mr. Alex Lee, and Mr. Harman Sandhu will be involved in screening and data extraction moving forward.

\section{Funding}

Funding comes from the Michael G. DeGroote Centre for Medicinal Cannabis Research, of which Dr. Mackillop is a co-director. Dr. MacKillop's contributions are supported in part by the Peter Boris Chair in Addictions Research.

\section{Authors' contributions}

$\mathrm{JH}$ contributed to concept development, protocol development, and was the lead author of the manuscript. TP contributed to protocol development and manuscript writing. AF contributed to protocol development and manuscript writing. CM contributed to concept development, protocol development, and manuscript writing. JM contributed to concept development, protocol development, and manuscript writing. All authors read and approved the final manuscript.

\section{Authors' information \\ $\mathrm{JH}$ is a registered nurse and graduate student in McMaster's Health Research Methodology Program in the Department of Health Research Methods, Evidence, and Impact. JH is a research assistant in the Peter Boris Centre for Addictions Research. TP is a graduate student in the Peter Boris Centre for Addictions Research. AF is a research coordinator at the Michael G. DeGroote Centre for Medicinal Cannabis Research and Peter Boris Centre for Addictions Research. CM is the lead psychiatrist at the McMaster University Student Wellness Centre, co-lead of the McMaster Student Mental Health and Well-being Strategy and an assistant clinical professor in the Department of Psychiatry. JM is the Peter Boris chair in Addictions Research, the director of the Peter Boris Centre for Addictions Research, the co-director of the Michael G. DeGroote Centre for Medicinal Cannabis Research, and a professor in the Department of Psychiatry \& Behavioral Neurosciences at McMaster University.}




\section{Consent for publication}

Not applicable.

\section{Competing interests}

Dr. Mackillop is a principal in BEAM Diagnostics, Inc. The authors have no other competing interests to declare.

\section{Publisher's Note}

Springer Nature remains neutral with regard to jurisdictional claims in published maps and institutional affiliations.

\section{Author details}

'Department of Health Research, Evidence, and Impact, McMaster University, Hamilton, Ontario, Canada. ${ }^{2}$ Michael G. De Groote Centre for Medicinal Cannabis Research, McMaster University/St. Joseph's Healthcare Hamilton, Hamilton, Ontario, Canada. ${ }^{3}$ Peter Boris Centre for Addictions Research, McMaster University/St. Joseph's Healthcare Hamilton, 100 West 5th Street, Hamilton, Ontario L8S 4L8, Canada. ${ }^{4}$ Department of Psychiatry and Behavioural Neurosciences, McMaster University, Hamilton, Ontario, Canada.

Received: 4 February 2018 Accepted: 10 July 2018

Published online: 25 July 2018

\section{References}

1. Schulenberg JE, Johnston LD, O'Malley PM, Bachman JG, Miech RA, Patrick ME. Monitoring the Future national survey results on drug use, 1975-2016: Volume II, college students and adults ages 19-55. Ann Arbor: Institute for Social Research, The University of Michigan. 2017.

2. Canadian centre on substance abuse cannabis. 2016. Library/CCSACanadian-Drug-Summary-Cannabis-2016-en.pdf. 25 October 2017. http:// www.ccdus.ca/Resource\%20Library/CCSA-Canadian-Drug-SummaryCannabis-2018-en.pdf.

3. National Intsitute on Drug Abuse. Most commonly used addictive drugs. 2016. https://www.drugabuse.gov/publications/media-guide/mostcommonly-used-addictive-drugs. 25 October 2017.

4. Statistics Canada. Canadian tobacco, alcohol and drugs survey: summary of results for 2013. 2015. healthycanadians.gc.ca/science-research-sciencesrecherches/data-donnees/ctads-ectad/summary-sommaire- 2013-eng.php. 14 September 2017

5. United Nations. What do we mean by "youth"? United Nations educational, scientific and cultural organization 2017. http://www.unesco.org/new/en/ social-and-human-sciences/themes/youth/youth-definition/. 4 December 2017.

6. Volkow ND, Compton WM, Weiss SR. Adverse health effects of marijuana use. N Engl J Med. 2014;371(9):879.

7. American college health association. American college health associationnational college health assessment II: Canadian reference group data report spring 2016. Hanover: American college health association; 2016.

8. American college health association. American college health associationnational college health assessment II: Candian reference group executive summary spring 2013. Hanover: American college health association; 2013

9. Gavin L, Mackay AP, Brown K, et al. Sexual and reproductive health of persons aged 10--24 years --- United States, 2002--2007. Morb Mortal Wkly Rep Surveill Summ. 2009;58(SS06):1-58.

10. Pope HG Jr, Gruber AJ, Hudson Jl, Huestis MA, Yurgelun-Todd D. Neuropsychological performance in long-term cannabis users. Arch Gen Psychiatry. 2001;58(10):909-15.

11. Broyd SJ, van Hell HH, Beale C, Yucel M, Solowij N. Acute and chronic effects of cannabinoids on human cognition-a systematic review. Biol Psychiatry. 2016;79(7):557-67.

12. Block Rl, O'Leary DS, Hichwa RD, Augustinack JC, Boles Ponto LL, Ghoneim MM, Arndt S, Hurtig RR, Watkins GL, Hall JA, Nathan PE, Andreasen NC. Effects of frequent marijuana use on memory-related regional cerebral blood flow. Pharmacol Biochem Behav. 2002;72(1-2):237-50.

13. Meier MH, Caspi A, Ambler A, Harrington H, Houts R, Keefe RS, McDonald K, Ward A, Poulton R, Moffitt TE. Persistent cannabis users show neuropsychological decline from childhood to midlife. Proc Natl Acad Sci U S A. 2012;109(40):E2657-64

14. Hall W. What has research over the past two decades revealed about the adverse health effects of recreational cannabis use? Addiction. 2015;110(1):19-35
15. Phillips KT, Phillips MM, Lalonde TL, Tormohlen KN. Marijuana use, craving, and academic motivation and performance among college students: an inthe-moment study. Addict Behav. 2015;47:42-7.

16. Arria AM, Caldeira KM, Bugbee BA, Vincent KB, O'Grady KE. Marijuana use trajectories during college predict health outcomes nine years postmatriculation. Drug Alcohol Depend. 2016;159:158-65.

17. Suerken CK, Reboussin BA, Egan KL, Sutfin EL, Wagoner KG, Spangler J, Wolfson M. Marijuana use trajectories and academic outcomes among college students. Drug Alcohol Depend. 2016;162:137-45.

18. Lev-Ran S, Le Foll B, McKenzie K, George TP, Rehm J. Cannabis use and cannabis use disorders among individuals with mental illness. Compr Psychiatry. 2013;54(6):589-98.

19. Gage SH, Hickman M, Zammit S. Association between cannabis and psychosis: epidemiologic evidence. Biol Psychiatry. 2016;79(7):549-56.

20. Lev-Ran S, Le Foll B, McKenzie K, George T, Rehm J. Marijuana use and marijuana use disorders among individuals with mental illness. Compr Psychiatry. 2013;54(4):589-98.

21. Horwood LJ, Fergusson DM, Coffey C, Patton GC, Tait R, Smart D, Letcher P, Silins E, Hutchinson DM. Cannabis and depression: an integrative data analysis of four Australasian cohorts. Drug Alcohol Depend. 2012;126(3):369-78.

22. Moore TH, Zammit S, Lingford-Hughes A, Barnes TR, Jones PB, Burke M, Lewis $\mathrm{G}$. Cannabis use and risk of psychotic or affective mental heath outcomes: a systematic review. Lancet. 2007;370(9584):319-28.

23. Kedzior KK, Laeber LT. A positive association between anxiety disorders and cannabis use or cannabis use disorders in the general population--a metaanalysis of 31 studies. BMC Psychiatry. 2014;14:136.

24. Borges G, Bagge CL, Orozco R. A literature review and meta-analyses of cannabis use and suicidality. J Affect Disord. 2016;195:63-74.

25. Boak A, Hamilton HA, Adlaf EM, Mann RE. Drug use among Ontario students, 1977-2017: Detailed findings from the Ontario Student Drug Use and Health Survey (OSDUHS) (CAMH Research Document Series No. 46). Toronto: Centre for Addiction and Mental Health. 2017.

26. Okaneku J, Vearrier D, McKeever RG, LaSala GS, Greenberg MI. Change in perceived risk associated with marijuana use in the United States from 2002 to 2012. Clin Toxicol (Phila). 2015;53(3):151-5.

27. Schulenberg JE, Johnston LD, O'Malley PM, Bachman JG, Miech RA and Patrick ME. Monitoring the Future national survey results on drug use, 1975-2016: Volume II, College students and adults ages 19-55. Ann Arbor: Institute for Social Research, The University of Michigan; 2017. http:// monitoringthefuture.org/pubs.html\#monographs.

28. Substance Abuse and Mental Health Services Administration. Results from the 2013 National Survey on Drug Use and Health: Summary of National Findings, NSDUH Series H-48, HHS Publication No. (SMA) 14-4863. Rockville, MD: Substance Abuse and Mental Health Services Administration. 2014. https://www.samhsa.gov/data/sites/default/files/NSDUHresultsPDFWHTML 2013/Web/NSDUHresults2013.pdf.

29. Berg CJ, Stratton E, Schauer GL, Lewis M, Wang Y, Windle M, Kegler M. Perceived harm, addictiveness, and social acceptability of tobacco products and marijuana among young adults: marijuana, hookah, and electronic cigarettes win. Subst Use Misuse. 2015;50(1):79-89.

30. American college health association. American college health associationnational college health assessment II: Undergraduate student reference group data report fall 2016. Hanover: American college health association; 2017.

31. Hunt J, Eisenberg D. Mental health problems and help-seeking behavior among college students. J Adolesc Health. 2010;46(1):3-10.

32. Gulliver A, Griffiths KM, Christensen $H$. Perceived barriers and facilitators to mental health help-seeking in young people: a systematic review. BMC Psychiatry. 2010;10:113.

33. Eisenberg D, Golberstein E, Gollust SE. Help-seeking and access to mental health care in a university student population. Med Care. 2007;45(7):594-601.

34. Moyer A, Finney JW, Swearingen CE, Vergun P. Brief interventions for alcohol problems: a meta-analytic review of controlled investigations in treatmentseeking and non-treatment-seeking populations. Addiction. 2002;97(3):279-92.

35. Tevyaw TO, Monti PM. Motivational enhancement and other brief interventions for adolescent substance abuse: foundations, applications and evaluations. Addiction. 2004;99(Suppl 2):63-75.

36. Miller WR, Rollnick S. Motivational interviewing: helping people change. New York: Guilford press; 2012.

37. Winters KC, Leitten W, Wagner E, O'Leary Tevyaw T. Use of brief interventions for drug abusing teenagers within a middle and high school setting. J Sch Health. 2007;77(4):196-206 
38. World Health Organization. The ASSIST-linked brief intervention for hazardous and harmful substance use: a manual for use in primary care. In: World health organization; 2010. http://apps.who.int/iris/bitstream/10665/ 44321/1/9789241599399 eng.pdf?ua=1.

39. Matua Raki. Screening, assessment and evaluation: alcohol and other drug, smoking and gambling. National Addiction Workforce Development 2012. https:/www.tepou.co.nz/uploads/files/resource-assets/screening-assessmentand-evaluation-aod-smoking-and-gambling.pdf. 15 September 2017.

40. Bien TH, Miller WR, Tonigan JS. Brief interventions for alcohol problems: a review. Addiction. 1993;88(3):315-35.

41. Arnett JJ. Emerging adulthood. A theory of development from the late teens through the twenties. Am Psychol. 2000;55(5):469-80.

42. Jensen CD, Cushing CC, Aylward BS, Craig JT, Sorell DM, Steele RG. Effectiveness of motivational interviewing interventions for adolescent substance use behavior change: a meta-analytic review. J Consult Clin Psychol. 2011;79(4):433-40.

43. Barnett E, Sussman S, Smith C, Rohrbach LA, Spruijt-Metz D. Motivational interviewing for adolescent substance use: a review of the literature. Addict Behav. 2012;37(12):1325-34.

44. Carney T, Myers BJ, Louw J, Okwundu Cl. Brief school-based interventions and behavioural outcomes for substance-using adolescents. Cochrane Database Syst Rev. 2016;1:CD008969.

45. Dennhardt AA, Murphy JG. Prevention and treatment of college student drug use: a review of the literature. Addict Behav. 2013;38(10):2607-18.

46. Gates PJ, Sabioni P, Copeland J, Le Foll B, Gowing L. Psychosocial interventions for cannabis use disorder. Cochrane Database Syst Rev. 2016;5: CD005336.

47. Hartman M, Sreeram P, Wilson MG. Rapid Synthesis: Identifying the impacts of cannabis legalization on youth, and the responses that can be taken by public institutions. Hamilton: McMaster Health Forum; 2017.

48. Higgins JPT and Green S. Cochrane handbook for systematic reviews of interventions Version 5.1.0. The Cochrane Collaboration. 2011. http://www. handbook.cochrane.org. 28 January 2017.

49. Sterne JA, Hernan MA, Reeves BC, Savovic J, Berkman ND, Viswanathan M, Henry D, Altman DG, Ansari MT, Boutron I, Carpenter JR, Chan AW, Churchil R, Deeks JJ, Hrobjartsson A, Kirkham J, Juni P, Loke YK, Pigott TD, Ramsay CR, Regidor D, Rothstein HR, Sandhu L, Santaguida PL, Schunemann HJ, Shea B, Shrier I, Tugwell P, Turner L, Valentine JC, Waddington H, Waters E, Wells GA, Whiting PF, Higgins JP. ROBINS-I: a tool for assessing risk of bias in non-randomised studies of interventions. BMJ. 2016;355:44919.

50. Cohen J. Statistical power analysis for the behavioral sciences. New York: Lawrence Earlbaum Associates; 1988.

51. Schünemann H, Brożek J, Guyatt G and Oxman AE. GRADE Handbook for grading quality of evidence and strength of recommendations. The GRADE working group. 2013. guidelinedevelopment.org/handbook. April 15, 2017.

52. Miake-Lye IM, Hempel S, Shanman R, Shekelle PG. What is an evidence map? A systematic review of published evidence maps and their definitions, methods, and products. Syst Rev. 2016;5(1):28.

Ready to submit your research? Choose BMC and benefit from:

- fast, convenient online submission

- thorough peer review by experienced researchers in your field

- rapid publication on acceptance

- support for research data, including large and complex data types

- gold Open Access which fosters wider collaboration and increased citations

- maximum visibility for your research: over $100 \mathrm{M}$ website views per year

At BMC, research is always in progress.

Learn more biomedcentral.com/submissions 